\title{
Investigating Upper-Division Students' Interpretations of the Divergence Theorem
}

\author{
James A. Huffman, ${ }^{1}$ Michael Vignal, ${ }^{1}$ and Bethany R. Wilcox ${ }^{1}$ \\ ${ }^{1}$ Department of Physics, University of Colorado, 390 UCB, Boulder, CO 80309
}

(Dated: May 2020)

The divergence theorem is an important mathematical tool used in many areas of physics, most notably, electricity and magnetism. Students have nominally encountered the divergence theorem in their math courses; however, its application in physics often differs meaningfully from its application in a pure math context. In this paper, we investigate physics students' understanding and interpretation of the divergence theorem through written responses to a brief two-part prompt which was designed to elicit separate responses for students' literal and qualitative interpretations of the divergence theorem. This differentiated between understanding the mathematical statement and being able to interpret its fundamental meaning. We found that students provided moderately successful literal interpretations but provided fewer meaningful qualitative interpretations. We also found that the ability to accurately translate the mathematical statement of the divergence theorem appeared to be a necessary, but not sufficient condition for being able to provide a meaningful interpretation.

\footnotetext{
2020 PERC Proceedings edited by Wolf, Bennett, and Frank; Peer-reviewed, doi.org/10.1119/perc.2020.pr.Huffman

Published by the American Association of Physics Teachers under a Creative Commons Attribution 4.0 license. Further distribution must maintain the cover page and attribution to the article's authors.
} 


\section{INTRODUCTION \& BACKGROUND}

Recent years have seen an increased interest in understanding students' use of mathematics in physics problem solving, particularly at the upper-division level where the necessary mathematical tools become more sophisticated [1]. For example, in the context of electricity and magnetism (E\&M), vector calculus becomes a crucial tool in the development of electromagnetic theory. In most physics programs, students have completed the calculus sequence along with a course on linear algebra and differential equations by the time they encounter E\&M at the upper-division level. This timeline ensures that students have been exposed to vector calculus in their mathematics courses before they encounter it in E\&M; however, the application of vector calculus to physics problem solving often differs in meaningful ways from the presentation in mathematics courses [1]. As such, it is important to understand what challenges arise for students when using vector calculus in the context of physics.

A small but growing body of literature has focused on students' difficulties with aspects of vector calculus, particularly around the divergence and curl of vector fields. For example, Bollen et al. [2] investigated students' difficulties with the divergence and curl in the context of understanding Maxwell's equations. They found that, while students showed significant proficiency at performing calculations involving the divergence and curl, students struggled to interpret graphical representations of vector fields with respect to their divergence and curl. They also found that students had difficulty articulating a robust conceptual description of the divergence and curl. In a later study, Bollen et al. [3] built on this earlier work to show that difficulties with the conceptual aspects of vector calculus operations can lead to improper understandings of Maxwell's equations.

Similar difficulties with conceptualizing the divergence and curl have been observed in other studies $[4,5]$. Additionally, Pepper et al. [5] documented students' difficulties when setting up and interpreting vector integrals in the context of, for example, Gauss' Law. They attributed many challenges they observed to students' difficulty conceptualizing these integrals as the 'sum of little bits.'

In this study, we contribute to this body of work by investigating students' interpretations of the divergence theorem. Mathematically, the divergence theorem is given as follows:

$$
\oiint \vec{v} \cdot d \vec{a}=\iiint(\vec{\nabla} \cdot \vec{v}) d \tau
$$

where $\vec{v}$ is an arbitrary vector field, and $d \vec{a}$ and $d \tau$ represent infinitesimal area and volume elements respectively [6]. The divergence theorem is a critical tool in electromagnetic theory as it is used to navigate between the integral and differential forms of several of Maxwell's equations, including Gauss's Law. Interpreting the meaning of this theorem requires a robust understanding of the divergence of a vector field and also
The divergence theorem has important applications in electrodynamic theory, and in particular, to Gauss's law. Given a vector field $\vec{v}$ and a closed surface $s$ which encloses the volume $\tau$, the divergence theorem can be stated as follows:

$$
\oiint \vec{v} \cdot d \vec{a}=\iiint(\vec{\nabla} \cdot \vec{v}) d \tau
$$

1. Translate the mathematical statement of the divergence theorem into words (i.e. without symbols).

2 . When applied to a physical vector field what does this mathematical statement mean qualitatively (i.e. how would you explain this to someone who has no experience with calculus)? If you are uncertain about the overall interpretation, describe the meaning of each integrand and comment on any element that you find confusing. Include any formulas or diagrams that you think will be helpful in your explanation.

FIG. 1. The research task as given to students. Note that the students were given space to show their work.

the integration of vector fields both over a surface and throughout a volume.

In this paper, we investigate how students make sense of the divergence theorem by both asking them to translate the theorem into words and interpret its meaning when applied to a physical vector field (the exact prompts used are shown in Fig. 1). We argue, pulling from the work of Redish and Kuo [7], that these two tasks are distinct, with the former addressing student recognition of the mathematical syntax of the divergence theorem and the latter targeting its use and meaning. In the following section, we describe the methods and context for this study (Sec. II). Section III presents our main findings and preliminary interpretations. Finally, Sec. IV discusses conclusions, limitations, and future work.

\section{CONTEXT \& METHODS}

Data for this study were collected in the first semester of the junior-level electricity and magnetism (E\&M) course at a large research institution in the central United States. The course is the first in a two course sequence and covers chapters 1-6 of Griffiths' text [6] and typically enrolls 70-100 physics and engineering physics majors. This course has a prerequisite of Calculus 3, which includes vector calculus, and by this point in the sequence, students will have also taken a differential equations course.

Data for the study consist of students' responses to a short research task (Fig. 1). The research task consisted of two prompts designed to elicit students' literal and qualitative interpretations of the divergence theorem. The task was laid out in two sections, as shown in Fig. 1. First, the prompt provided a brief statement including some context about the background of the divergence theorem as well as a succinct re-statement of the theorem at the top of the task sheet. In stating the divergence theorem, the prompt provided equation (1) and included a definition of all relevant variables.

A number of considerations were made in the design 
of the task and prompts. In the first section of the task, we needed to balance providing a clear and unambiguous statement of the divergence theorem without giving too much information in the question prompt. Notation choices in the prompt were consistent with Griffiths' text and our goals for the types of justifications we were interested in eliciting. For example, we chose to use $\vec{v} \cdot d \vec{a}$ instead of $(\vec{v} \cdot \hat{n}) d a$ in order to make the direction of $d \vec{a}$ less explicit. By doing so, we would be able to determine whether students would (or could) spontaneously address the directionality of $d \vec{a}$. Another notation choice was the use of $d \tau$ as the volume element. This was chosen in order not to confuse $d v$ with $\vec{v}$, our vector field label, and to also make the meaning of $d \tau$ less transparent. Again, this choice was to provide insight into how students reasoned around, for example, the shape of the volume being integrated over.

The wording of the task prompts was arrived at through iterative discussion and refinement by the authors. Each prompt was worded with the types of responses we were looking to elicit in mind. For the translate prompt (part 1. in Fig. 1), we wanted students to provide a direct 'translation' of the divergence Theorem in order to see how they would interpret the nuances of the mathematical syntax that are present. Additionally, we wanted to disambiguate difficulties students might be having with interpreting the divergence theorem from those they might have parsing the mathematical syntax. The specific language of note was the operative verb 'translate', which most succinctly describes the intended task; several revisions were made before arriving at this word choice. Finally, we wanted to be as explicit as possible that responses should not contain direct restatements of the original mathematical syntax.

For the interpretation prompt (part 2. in Fig. 1), we sought to elicit students' interpretations of the divergence theorem apart from its purely mathematical meaning. Mindful that the divergence theorem can be applied to many different physical vector fields, we chose not to use a specific example of vector field (e.g., the electric field), instead allowing the students to provide their own physical context. In doing so, we hoped to glean insight into any intuitive approaches that students might take in understanding the divergence theorem, and whether these approaches would be grounded in a particular physical context for which they might have more intuition.

Students completed the research task during the first $\sim 5$ minutes of class during the second week of the 15 week course. The relevant sections of Griffiths' textbook were assigned as reading for the day that students completed this activity; however, students had not yet had any explicit instruction on the divergence theorem or Gauss' law as part of the course. Students had previously completed readings for Chapter 1 of Griffiths' textbook, which includes a section that covers the divergence theorem in some detail. In total, 81 students were enrolled in the course, and we received 60 responses to the research task, a response rate of $74 \%$.
We coded student responses using a hybrid emergentaxial coding process [8]. Since the meanings of the symbols in the divergence theorem are unambiguous, we performed an axial coding for the translation prompt in which we characterized how accurately students translated the symbols in the theorem into words: we identified five aspects of the translation, the left hand side (LHS) and right hand side (RHS) integrands, the LHS and RHS integrals, and how the bounds of the LHS and RHS integrals relate to one another.

Student responses were coded as accurate, somewhat accurate, or inaccurate based on how well students translated the divergence theorem for each of these aspects: an accurate response included most elements of the translation and few if any omissions or incorrect statements; a somewhat accurate response included some correct (or appropriate) elements of the translation with some incorrect statements or omissions; and inaccurate responses included few or no elements of the translation or more significant omissions or incorrect statements (see Sec. III for examples of prompts coded in each of these categories). Non-responses were coded separately. We then performed an emergent coding of student responses to identify common themes, paying special attention to common inaccuracies in student translations.

A similar process was repeated for the students' qualitative interpretations of the divergence theorem that were given in response to prompt 2 . In this coding, we again looked at the same five aspects of the divergence theorem-LHS and RHS integrands and integrals, and how the LHS and RHS bounds are connected - though we did not code student responses using a priori codes because the nature of the question (interpretation) is not conducive to a priori coding. Instead, we used iterative emergent coding to identify themes in the student responses, then we characterized these responses as accurate, somewhat accurate, and inaccurate, taking into account the type of interpretation that the students gave. For example, some students identified the LHS integrand as flux, while others talked about the dot product between the vector field and a surface element. Both of these ideas are legitimate ways of thinking about the LHS integrad, though with either line of thinking we still saw many inaccuracies in student reasoning.

Codes were initially developed by J.A.H. and iteratively refined until they were agreed upon by all of the authors. The following section describes the results of this coding, as well as some of the themes that emerged.

\section{RESULTS \& INTERPRETATIONS}

The elements that distinguished accurate, somewhat accurate and inaccurate responses emerged during the iterative coding process and were agreed upon by the authors. An example of a close to accurate response (coded as accurate) to the translate prompt was:

"The surface integral of a vector field over a closed surface is equal to the volume integral 
of the divergence of the vector field over the volume enclosed by the surface."

This response included comments about the connection between the LHS and RHS bounds, the closed nature of the surface, the dimensionality (i.e., surface vs. volume) of each integral, and identified the divergence, while omitting details relating to $d \vec{a}$ and the dot product with $\vec{v}$.

An example of a somewhat accurate response to the translate prompt was:

"The surface integral of the field is equal to

the volume integral of the divergence of that field."

While this response similarly omitted elements about the dot product, it was coded as somewhat accurate because it also omitted comments about the connection between LHS and RHS bounds, the closed nature of the surface, and the vector nature of the field.

Responses to the translate prompt that were coded as inaccurate response were diverse. For example:

"The 'squishiness' of a vector field through a surface? Honestly not too sure ..."

This response suggests the student did not have a clear starting point for a translation and did not address any of the axial codes we identified. A different response that was also coded as inaccurate was:

"The derivative of vector field $\vec{v}$ in a surface

is the function of the vector field $\vec{v}$ in a cube (volume)"

Although this response included several of the identified elements of the translation, several incorrect statements are made and the response does not provide a clear translation of the divergence.

Table I provides the breakdown of students responses for both prompts in Fig. 2 broken down by the LHS and RHS of Eqn. 1. Table I shows that, of 60 students, 18 provided an accurate translation of the LHS (i.e., the flux side), while only 13 provided an accurate interpretation.

Students responses with respect to the right hand side show a similar pattern, with 31 students providing an accurate translation for the RHS and only 7 providing an accurate interpretation. This trend shows that, overall, students had more success translating the divergence theorem than interpreting it. This trend is further reflected in the number of students who gave non-responses to each prompt. A non-response occurred when a student

TABLE I. Accuracy of responses broken down by side of the divergence theorem as stated in Eqn. 1 for prompt 1 (translate) and prompt 2 (interpret). Abbreviations in the table are defined as follows: ACC - Accurate, SWA - Somewhat Accurate, INA - Inaccurate, NR - non-response.

\begin{tabular}{|c|c|c|c|c|}
\hline & \multicolumn{2}{|c|}{ Prompt 1} & \multicolumn{2}{|c|}{ Prompt 2} \\
\hline Code & LHS & RHS & LHS & RHS \\
\hline $\mathrm{ACC}$ & 18 & 31 & 13 & 7 \\
\hline SWA & 21 & 13 & 17 & 18 \\
\hline INA & 20 & 15 & 17 & 22 \\
\hline NR & 1 & 1 & 13 & 13 \\
\hline
\end{tabular}
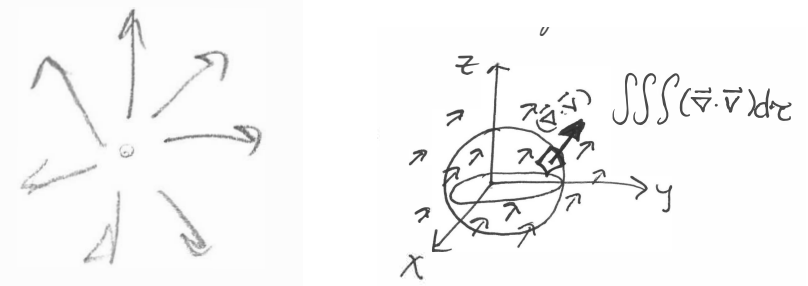

FIG. 2. Two depictions of divergence by students. The left image shows the more common depiction of divergence as a spread from a point, while the right image shows a student who depicted divergence as a sum of the field itself.

either left the question blank or directly stated 'I do not know.' Only one student provided a non-response to the translation prompt while a full 13 did so for the interpretation prompt. While it is possible that some students did not have time to complete the second prompt, our observations during the administration of the research task suggests that all students had sufficient time to complete the first prompt and move on to the second, and the majority of students had stopped working by the time they were asked to hand in their work. Thus, the increase in non-responses for prompt 2 suggests that students perceived this part of the task as more difficult.

Notably, while more than half of students accurately translated the RHS of Eqn. 1, only roughly a tenth were successful in interpreting it. Comments left by several students suggest possible contributing factors in their difficulty interpreting the RHS, including difficulty visualizing divergence, which was reflected in some of the drawings students provided to depict divergence. These drawings most commonly depicted divergence as a spread away from a single point in space, as shown in the left image in Fig. 2. Alternately, some students included drawings that seemed more in line with representing flux through a surface without any clear divergence in the drawn field. An example of such a diagram is given in the right image in Fig. 2.

Table II summarizes the categorization for response accuracy across both prompts, and two notable patterns emerge. Of the students who provided an accurate interpretation of the divergence theorem all but one gave accurate translation. However, the reverse was not true - giving an accurate translation was not strongly correlated with providing an accurate interpretation. This suggests that a clear and complete understanding of the mathematical syntax of the divergence theorem is a necessary, but not sufficient, condition for providing a robust interpretation of its meaning.

In addition to investigating the accuracy of students students responses as a whole, we were also interested in capturing students ideas around individual elements of the divergence theorem. For example, as discussed above and consistent with prior literature described in Sec. I, the divergence itself may be difficult for students to conceptualize. As such, we coded how students inter- 
TABLE II. Comparison of students' responses with respect to the accuracy of their response to prompt 1 (translate) and prompt 2 (interpret) responses. Rows indicate the code for prompt one, while columns indicate the code for prompt two. Abbreviations in the table are defined as follows: ACC - Accurate, SWA - Somewhat Accurate, INA - Inaccurate, NR non-response. $(\mathrm{n}=60)$

\begin{tabular}{llllll}
\hline \hline & \multicolumn{5}{c}{ Prompt 2 } \\
Prompt 1 & ACC & SWA & INA & NR & Total \\
\hline ACC & 8 & 7 & 6 & 2 & 23 \\
SWA & 1 & 13 & 7 & 5 & 26 \\
INA & 0 & 2 & 3 & 5 & 10 \\
NR & 0 & 0 & 0 & 1 & 1 \\
\hline Total & 9 & 22 & 16 & 13 & 60 \\
\hline \hline
\end{tabular}

preted the divergence independently. A total of 32 students provided a clear interpretation of the divergence in their response to prompt 2. Common ideas in students responses included (but were not limited to) that the divergence represented: the spread of the field $(N=8)$, the location of sources $(N=7)$, how much the field diverges $(N=6)$, the amount of the field that 'passes through' a volume $(N=5)$, and the 'change' in the field $(N=3)$. With the exception of the 'source' interpretation consistent with the typical expert response, most of these ideas are not 'incorrect,' but rather incomplete or underdeveloped. However, they represent important elements from which a more robust interpretation could be built.

It is worth noting that the interpretation that the divergence represents the 'spread' in the vector field is the most common interpretation, and is also very close to an expert like response. Griffiths himself describes the divergence as the amount that the field 'spreads from a point.' However, the addition of 'from a point' is crucial to making this interpretation unambiguous, particularly in the context of understanding the difference between a diverging field and one that just appears to spread out. For example, the field from a point charge appears to be spreading out at all points in space despite the fact that this field only has non-zero divergence at the location of the actual charge. We hypothesize that instructors (both in math and physics courses) are not always explicit in their description of divergence as the spread of the field from a point, and our data suggest this may result in students failing to recognize the importance of this subtlety.

\section{CONCLUSIONS}

In this paper, We investigated student understanding of a sophisticated mathematical tool - the divergence theorem - after instruction in math courses but prior to instruction in an upper-division E\&M course. We asked students in the second weeks of this E\&M course to translate the divergence theorem into words and then provide an interpretation of the theorem. We evaluated the research prompt responses to identify emergent themes in students understanding of the mathematical syntax of the divergence theorem as well as their interpretations of the theorem's conceptual meaning.

We found that our students were significantly more successful in translating the divergence theorem into words than they were at providing comprehensive and accurate interpretations of the theorem. Moreover, $48 \%$ of the population simply wrote 'I do not know' and/or did not provide any interpretation at all. This finding was especially stark for the RHS of the theorem, where students can readily identify divergence and the volume integral, but where they struggled to provide accurate interpretations. Additionally, we found that students provided a variety of interpretations of the divergence itself, many of which were at least partially correct, if not complete.

Our findings have implications for physics instructors teaching content requiring the use of the divergence theorem. These results suggest that, while students have seen the divergence theorem in their math courses and likely understand its underlying syntax, instructors should not assume that physics students gain the qualitative understanding of the divergence theorem from their math courses that they will need to employ the divergence theorem effectively in physics. For this reason, we anticipate that many physics instructors will need to include some instruction around the divergence theorem in their course. Moreover, this instruction may need to be accompanied by more explicit discussion of the qualitative meaning of the divergence itself. We caution against the use of imprecise terms like 'spread' in favor of promoting more unambiguous interpretations relating divergence to sources and sinks of the vector field.

One important limitation of this work relates to the student population used for the study. The institution at which the study took place is a highly selective research institution with a non-representative student body. As such, the trends we report here are unlikely to be comprehensive, and in particular, students from a different student population may encounter the difficulties described here more frequently or may encounter additional issues not observed in this population. In future work, we will extend this study through collection of data through student interviews and pre/post testing. In particular, we are interested in investigating how students understanding of the divergence theorem changes after instruction in the context of a physics course. Future work will also include development of a tutorial based on these findings to help students develop a robust interpretation of the divergence theorem. Possible extensions of this work could include a complementary investigation of student understanding of Stokes' theorem.

\section{ACKNOWLEDGEMENTS}

We would like to thank N. Crossette for his feedback in designing the research task, as well as the members of PER@C for their support. This work was supported by the CU Department of Physics. 
[1] Marcos D Caballero, Bethany R Wilcox, Leanne Doughty, and Steven J Pollock, 'Unpacking students' use of mathematics in upper-division physics: where do we go from here?" European Journal of Physics 36, 065004 (2015).

[2] Laurens Bollen, Paul van Kampen, and Mieke De Cock, "Students' difficulties with vector calculus in electrodynamics," Phys. Rev. ST Phys. Educ. Res. 11, 020129 (2015).

[3] Laurens Bollen, Paul van Kampen, Charles Baily, and Mieke De Cock, "Qualitative investigation into students' use of divergence and curl in electromagnetism," Phys. Rev. Phys. Educ. Res. 12, 020134 (2016).

[4] Charles Baily and Cecilia Astolfi, "Student reasoning about the divergence of a vector field," in Physics Education Research Conference 2014, PER Conference (Min- neapolis, MN, 2014) pp. 31-34.

[5] Rachel E. Pepper, Stephanie V. Chasteen, Steven J. Pollock, and Katherine K. Perkins, "Observations on student difficulties with mathematics in upper-division electricity and magnetism," Phys. Rev. ST Phys. Educ. Res. 8, 010111 (2012).

[6] David J. Griffiths, Introduction to electrodynamics (Prentice Hall, 1999).

[7] Edward F Redish and Eric Kuo, "Language of physics, language of math: Disciplinary culture and dynamic epistemology," Science \& Education 24, 561-590 (2015).

[8] Steven E. Stemler, "Content analysis," Emerging Trends in the Social and Behavioral Sciences: An Interdisciplinary, Searchable, and Linkable Resource , 1-14 (2015). 